\title{
Computer Simulation of Final-Stage Sintering: II, Influence of Initial Pore Size
}

\author{
I-Wei Chen, * Gregory N. Hassold, *** and David J. Srolovitz \\ Department of Materials Science and Engineering, University of Michigan, \\ Ann Arbor, Michigan 48109-2136
}

\begin{abstract}
A two-dimensional Monte Carlo simulation procedure has been used to investigate the effect of the initial pore size on the microstructural evolution and the kinetics of final-stage sintering. The sintering time scales with $r_{0}^{4} / D_{g b}$ and the grain-growth time scales with $r_{0}^{2} / D_{m}$. Pores are found to effectively pin the grain boundaries from the beginning of final-stage sintering at a porosity of $\Phi=0.09$ until $\Phi=0.03$. For $\Phi \leq 0.03$, the remaining pores do not effectively retard grain-boundary migration and normal grain growth occurs. Small pores were found to be less effective at retarding grain growth than expected on the basis of a simple graingrowth pinning model. The mean pore size was found to be nearly constant throughout the simulations. [Key words: sintering, computers, theory, grain growth, microstructure.]
\end{abstract}

\section{Introduction}

I NHE preceding paper, ${ }^{1}$ hereafter referred to as part I, we have described a two-dimensional model for final-stage sintering and performed computer simulations using Monte Carlo techniques. In this model, both grains and pores are mapped onto a regular lattice and the resulting microstructure evolves in a self-consistent manner via grain growth and pore shrinkage, as dictated by the energetics and the kinetics of the system. By an appropriate choice of the nearest-neighbor interactions, grain-boundary and surface energies may be assigned separately. Similarly, the rate constants associated with grain-boundary diffusivity, surface diffusivity, and grainboundary mobility may also be independently assigned. The simulated microstructure bears close resemblance to those observed in cross sections of experimental samples during final-stage sintering. Features such as pore shrinkage, grain growth, pore migration, pore breakaway, and pore reattachment are observed in the simulations. It is also found that the sintering rate decreases monotonically with time and that it scales linearly with grain-boundary diffusivity - although deviations from the equilibrium shape of pores can slow down sintering somewhat.

Although the dependence of the sintering rate on grainboundary diffusivity is important and self-evident, it is also of great interest to explore the interrelationship between microstructural parameters, such as pore size, grain size, and porosity. In particular, it would be desirable to establish a quantitative correlation between the initial microstructure

T. M. Shaw - contributing editor

\footnotetext{
Manuscript No. 197911. Received December 15, 1989; approved June 14, 1990.

Presented at the 91st Annual Meeting of the American Ceramic Society, Indianapolis, IN, April 23-27, 1989 (Basic Science Division, Paper No. 63B-89).

Supported by the U.S. Department of Energy (BES), Grant No. DEFG02-87ER 45302 (IWC) and the Univeristy of Michigan (GNH and DJS). * Member, American Ceramic Society.

*Present address: Department of Science and Mathematics, GMI Engineering and Management Institute, Flint, MI 48504.
}

and the subsequent evolution. The Monte Carlo simulation method developed here is well suited for such a study. By varying the specified microstructure at the beginning of the simulation, the evolution of the microstructure can be continuously monitored. Moreover, by performing a sufficient number of Monte Carlo simulations, a large statistical sample can be obtained. In this way, both the averages and fluctuations of microstructural features, as well as their relation to each other and to the initial microstructures, can be quantitatively evaluated.

The onset of final-stage sintering is marked by the disappearance of the continuous pore channels (i.e., open porosity) in three dimensions. ${ }^{2}$ If these pore channels are distributed uniformly along all of the three-grain junctions, they become unstable via Rayleigh instability ${ }^{3}$ at a characteristic porosity of $\Phi_{0} \cong 0.09 .^{1}$ Pores of lenticular shape then form and are distributed along grain boundaries. At this porosity, we have found that the grain boundaries in two dimensions eventually become pinned, and a stable microstructure results unless pore shrinkage is allowed. Thus, the initial microstructure at the onset of final-stage sintering, in two dimensions (at least), appears to be determined by the pore size. The emphasis of this study is thus on the effect of the initial pore size. In particular, we will attempt to establish kinetic scaling laws that relate the different microstructural parameters.

\section{Simulation Procedure}

The basic model and the Monte Carlo methods used in the present study have been described in part I. Therefore, only a brief outline is provided here. The microstructure is represented by mapping the grain and pore microstructure onto a discrete, triangular lattice with a number (which we shall call a spin value) assigned to each site to represent grain orientations and pores. The energetics of the system is such that only nearest-neighbor interactions are considered. Pairs of unlike spins have higher energies than pairs of the same spin so that the grain-boundary energy, $E_{g b}$, is both finite and positive. In a similar way, a surface energy, $E_{s}$, can be introduced. A Monte Carlo procedure is conducted such that spin changes are allowed if the total energy of the system is lowered. ${ }^{4}$ The overall kinetics of this model can be shown to be equivalent to the kinetics dictated by absolute rate theory. ${ }^{4}$ (See also the last paragraph of Section II (4) in part I). In addition, vacancy diffusion is allowed by spin exchanges involving both grain sites and pore sites (i.e., "vacancies"). To permanently remove a vacancy (as is required for pore shrinkage), a vacancy from a pore is allowed to diffuse randomly along the grain boundary and is removed only if it has reached another pore (see part I for details). Thus, the grain-boundary diffusivity, $D_{g b}$ (which controls pore removal), surface diffusivity, $D_{s}$ (which controls pore shape), and grain-boundary mobility, $D_{m}$ (which controls grain growth), may be independently adjusted. The combination of these independent kinetic parameters and the interfacial (grain-boundary and surface) energies allows us to reasonably model the kinetic and thermodynamic aspects of final-stage sintering. 
The basic time unit in the present simulation is called one Monte-Carlo step (MCS) and corresponds to a number of spin change attempts equal to the number of sites in the lattice. Typical runs reported in this study vary between $10^{4}$ and $10^{7}$ MCS. Multiple runs (at least five) are performed for each set of parameters to obtain data without excessive statistical fluctuations. The length scale we will use in this study is that of the lattice spacing. Typically, the size of the entire triangular lattice is 40000 sites, where each site may be regarded as a microscopic material volume undergoing kinetic processes.

The initial random microstructure containing grains and pores was prepared to a specified initial porosity and pore size by two steps. First, a random state of lattice spins was "quenched" to a low temperature and then allowed to evolve (grain growth) into a polycrystal with a grain size which is comparable to the desired pore size. Second, a certain fraction of the grains in the above polycrystal were reassigned as pores (at a pre-selected porosity) and then allowed to evolve, without pore shrinkage, until the grains grew to the point that they were completely pinned by the pores. The initial microstructures used in the present study had an average linear pore size ranging from 1.8 to 7.1 , but all had an initial porosity of roughly 0.09 . The latter value was chosen because the Rayleigh instability, which marks the onset of final-stage sintering, occurs near this critical value of the porosity. ${ }^{1}$

\section{Results}

\section{(1) Microstructure}

Figures 1 and 2 compare instantaneous pictures of the simulated microstructures at various times. The initial porosity is approximately 0.095 in both cases but their initial average pore size $\left(r_{0}\right)$ differ by a factor of $3.4\left(r_{0}\right.$ is 2.2 in Fig. 1 and 7.5 in Fig. 2).

It is obvious that the two initial microstructures have different initial and final grain sizes; the one with finer pores has finer initial grains. This finer microstructure, shown as Fig. 1, sinters faster. For example, at $t=10^{5} \mathrm{MCS}$, the porosity is reduced to 0.014 in Fig. 1 but remains at 0.090 in Fig. 2 . At this time, however, the grain size of Fig. 1 has increased considerably (to a grain size nearly equal to that of Fig. 2).

Despite the apparently different rates of sintering and grain growth, all the microstructures in both Figs. 1 and 2 appear reasonably homogeneous and self-similar, except for the different sizes and number of pores and grains. Thus, it is reasonable to compare their sintering and grain-growth kinetics.

\section{(2) Dependence of Sintering and Grain Growth on Initial Pore Size}

To demonstrate the pore-size dependence of the shrinkage kinetics, we plot porosity, $\Phi$, versus time in Fig. 3 for simula-

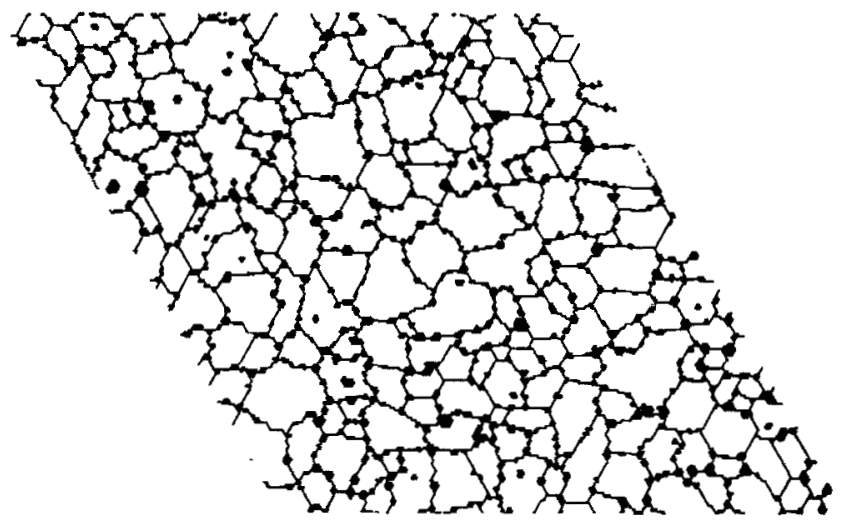

(A)

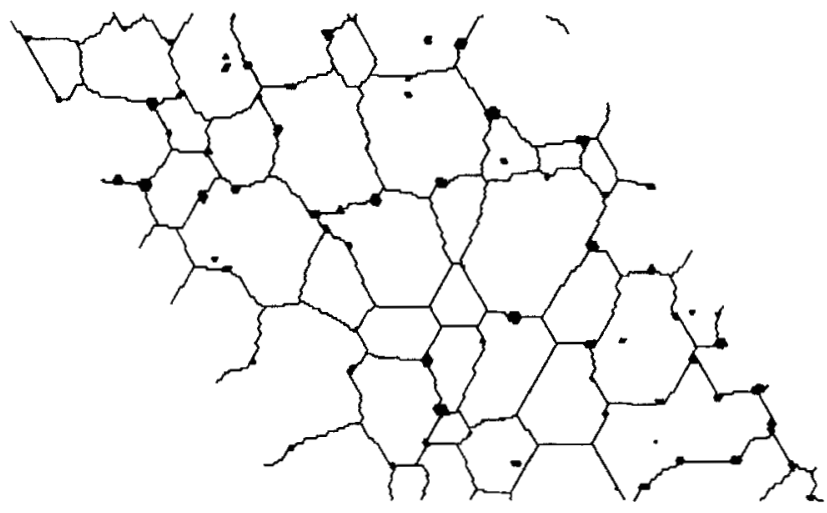

(C)

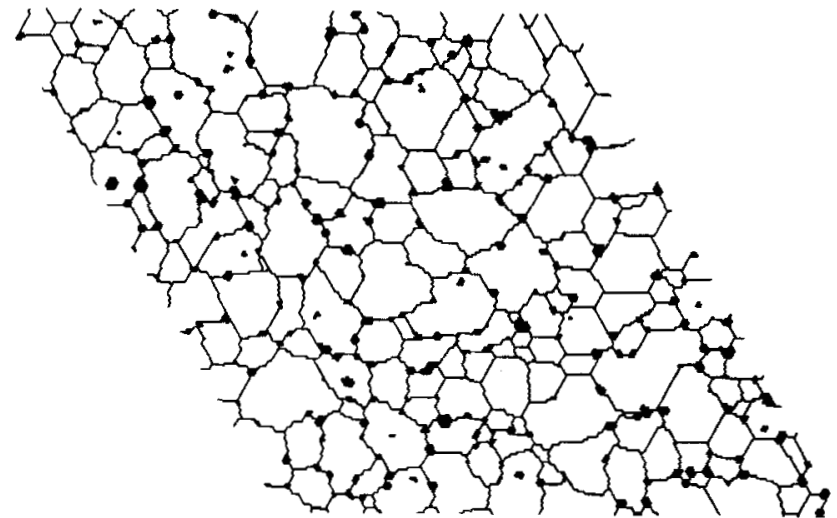

(B)

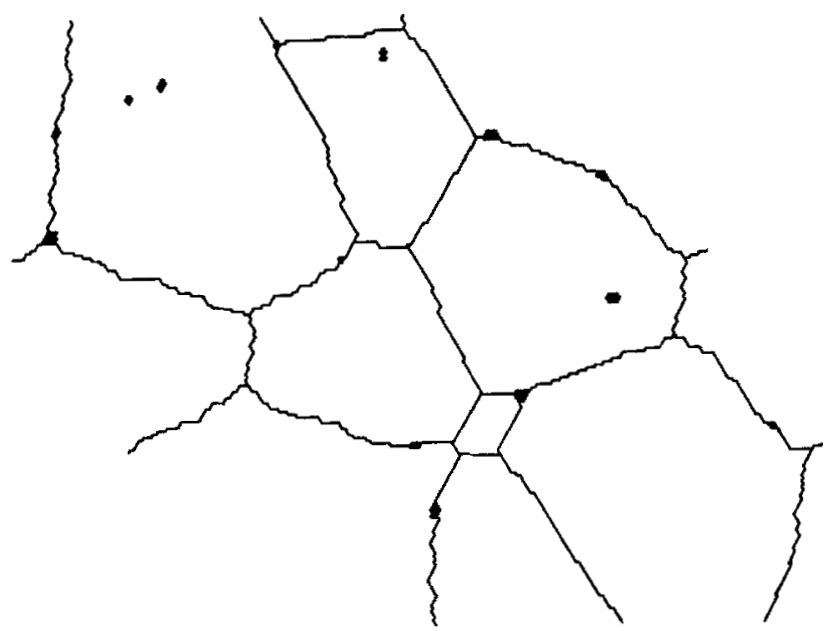

(D)

Fig. 1. Microstructural evolution of a porous polycrystalline solid undergoing final-stage sintering where $D_{g b} / D_{m}=0.1$ and $D_{s} / D_{m}=$ 1. Initial porosity, pore size, and grain size were $9.3 \%, 2.2$, and 14.2 , respectively. ((A) $1,(\mathrm{~B}) 10^{4}$, (C) $10^{5}$, and (D) $10^{6} \mathrm{MCS}$.) 


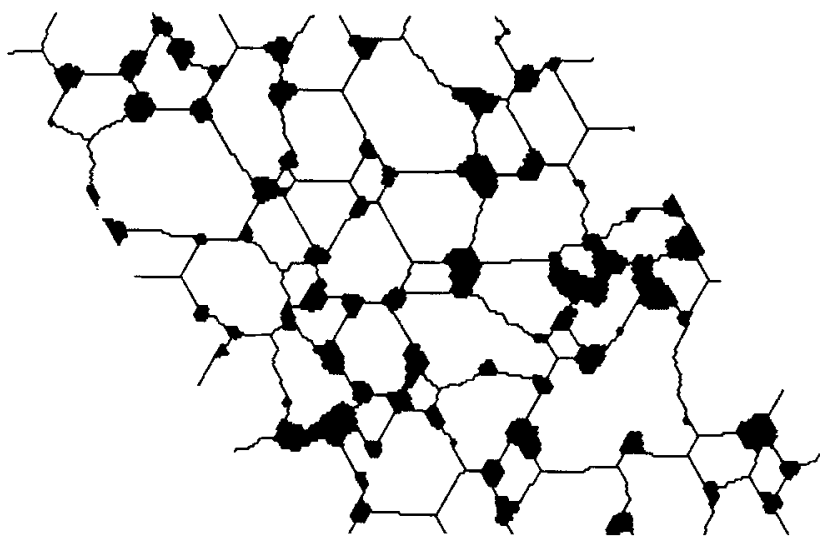

(A)

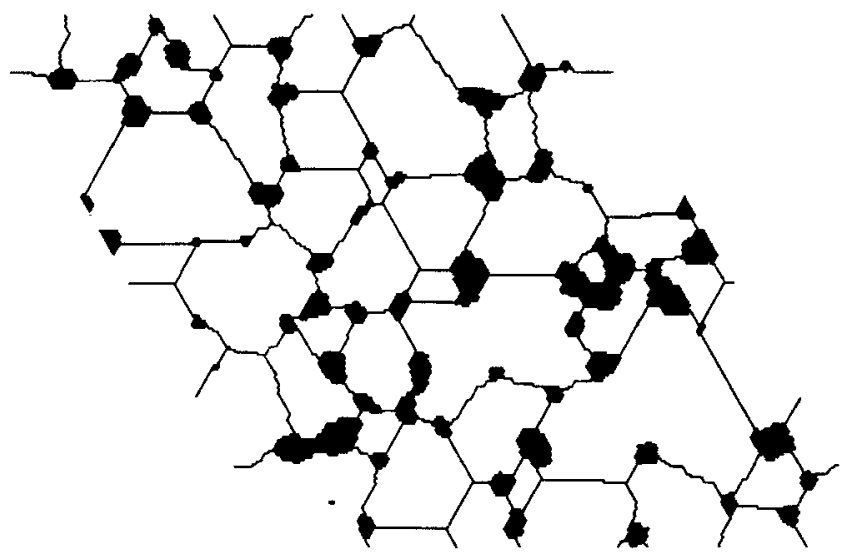

(C)

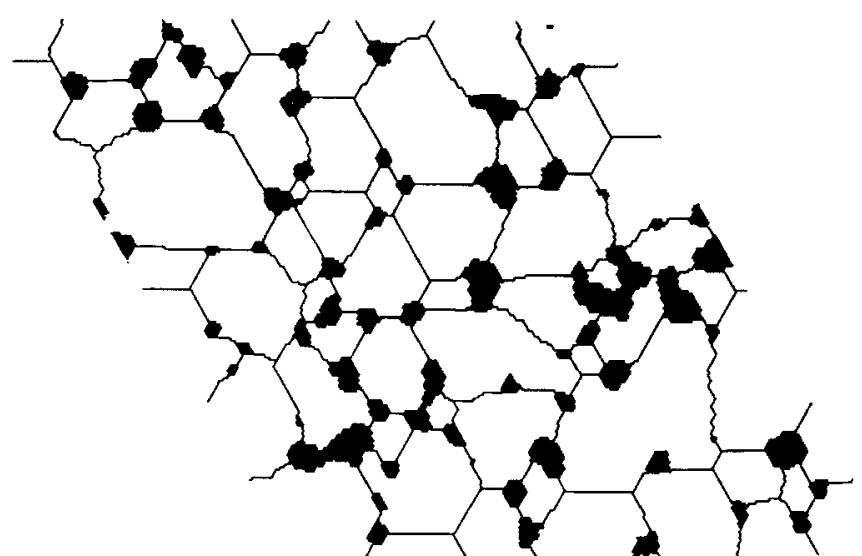

(B)

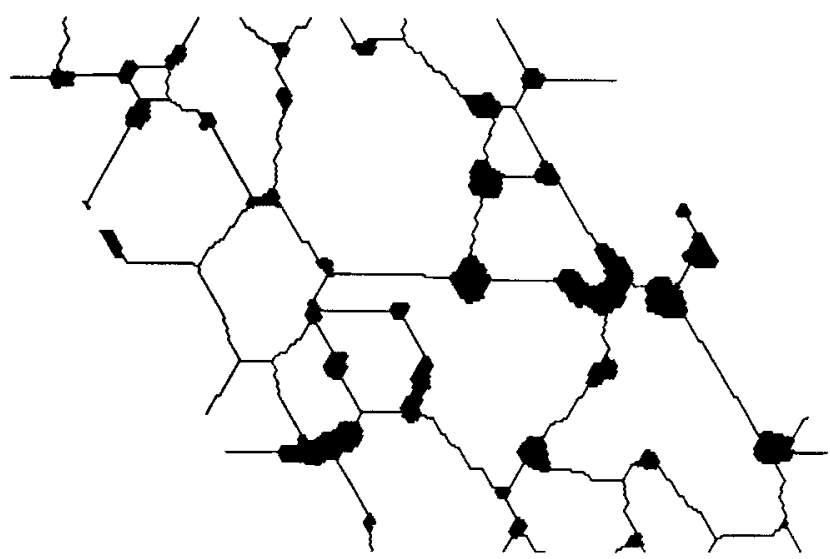

(D)

Fig. 2. Microstructual evolution of a porous polycrystalline solid undergoing final-stage sintering where $D_{g b} / D_{m}=0.1$ and $D_{s} / D_{m}=$ 1 . Initial porosity, pore size, and grain size were $10.0 \%, 7.5$, and 25.3 , respectively. ((A) 1, (B) $10^{4},(C) 10^{5}$, and (D) $10^{6} \mathrm{MCS}$.)

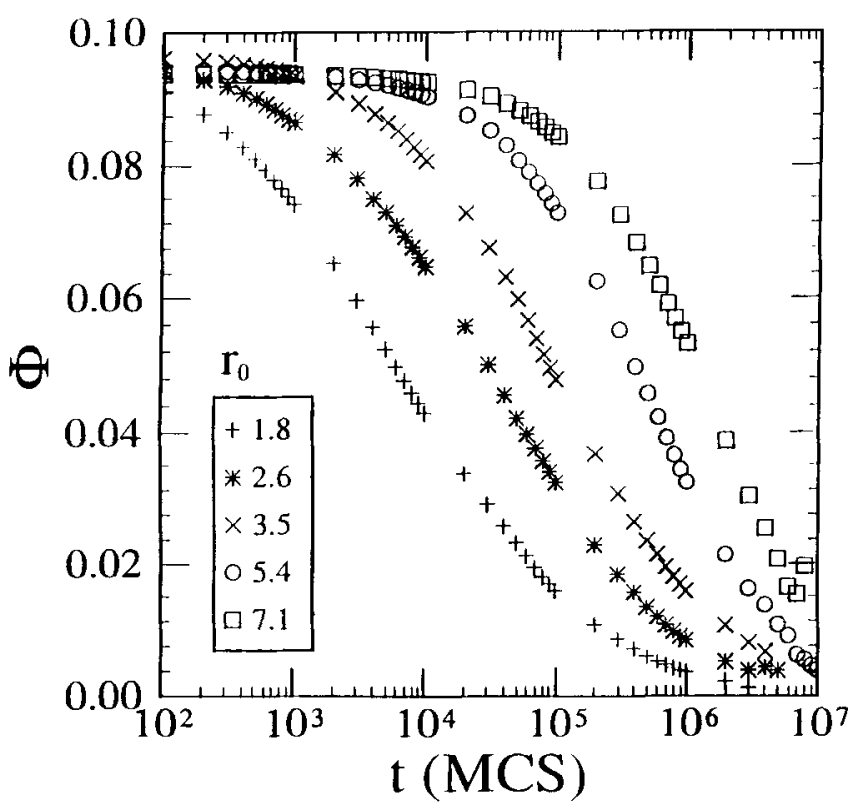

Fig. 3. Dependence of the porosity evolution kinetics on the initial pore size $r_{0}$ where $D_{g b} / D_{m}=0.1$ and $D_{s} / D_{m}=1$. tions with five different initial pore sizes in the range $1.8 \leq r_{0} \leq 7.1$. Although all the data follow the characteristic sigmoidal curve noted for shrinkage kinetics, they are displaced by more than 2 orders of magnitude in time, reflecting the strong dependence of the sintering rate on initial pore size.

The dependence of the grain growth rate on the initial pore size is indicated in Fig. 4 where we plot grain size, $R$, versus time for the same set of initial pore sizes considered in Fig. 3. The initial grain size increases monotonically with the initial pore size. However, as sintering proceeds, the branch with the smallest initial grains rises the fastest, and it eventually overtakes the one with the largest initial grain size. Indeed, for $t \geq 10^{6} \mathrm{MCS}$, the ordering of the grain size with pore size is just the opposite of that of the initial pore size. The reversal of this grain size ordering is a consequence of the faster shrinkage kinetics associated with the smallest pores. To account for the effect of porosity, the data from Figs. 3 and 4 are replotted as $R$ vs $\rho$, where $\rho=1-\Phi$ is the density. As shown in Fig. 5, the grain size grows slowly until porosity nearly disappears. It is now clear that, when microstructures are compared at a constant porosity, the grain size increases monotonically with the initial pore size.

We have attempted a renormalization of the data in Figs. 3 and 4 by taking into account the pore-size dependence. The best fit of these data into a single curve can be accomplished by normalizing $t$ by $r_{0}^{4}$ and $r_{0}^{2}$, in the case of $\Phi$ and $R$, respectively. In addition, the grain size is normalized by $R_{0}$. The results are shown in Figs. 6 and 7. Together these plots clearly demonstrate the common kinetic features of sintering and grain growth among all the microstructures studied, once the effects of inital pore size are properly considered. 


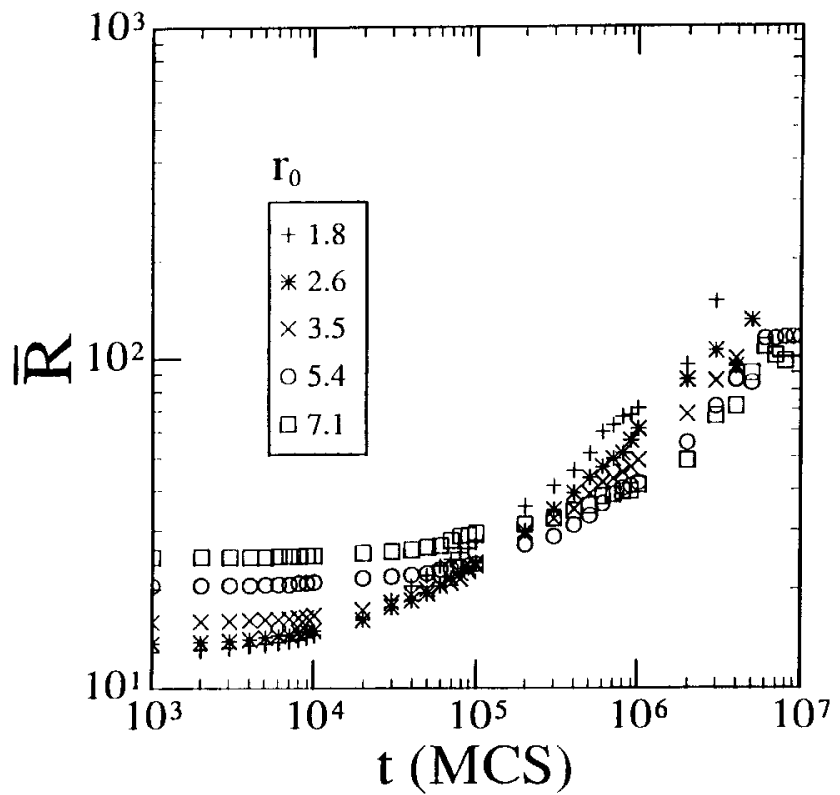

Fig. 4. Dependence of the mean grain-size evolution kinetics on the initial pore size, $r_{0}$, where $D_{g b} / D_{m}=0.1$ and $D_{s} / D_{m}=1$.

One might expect that a unique relation also exists for the normalized grain size and porosity. We have tested this hypothesis by replotting Fig. 5 in the form of $R / R_{0}$ vs $\Phi / \Phi_{0}$, as shown in Fig. 8. Although the curves exhibit a common trend, the scatter of the data in the intermediate portion is quite large. In the normalized coordinate, the microstructures with larger initial pore size show grain sizes which are relatively larger during sintering. We shall return to this point later.

\section{(3) Kinetics of Pore-Size and Grain-Size Evolution}

Individual pores are expected to shrink during sintering. However, pore "lifetimes" may vary widely depending on their initial size. This is shown in Figs. 1 and 2, in which some smaller pores disappear soon, whereas others remain much longer. To illustrate this, we plot the pore radius, $r$, versus time for eight individual pores during the course of a single simulation (Fig. 9). Clearly, larger pores have a much longer

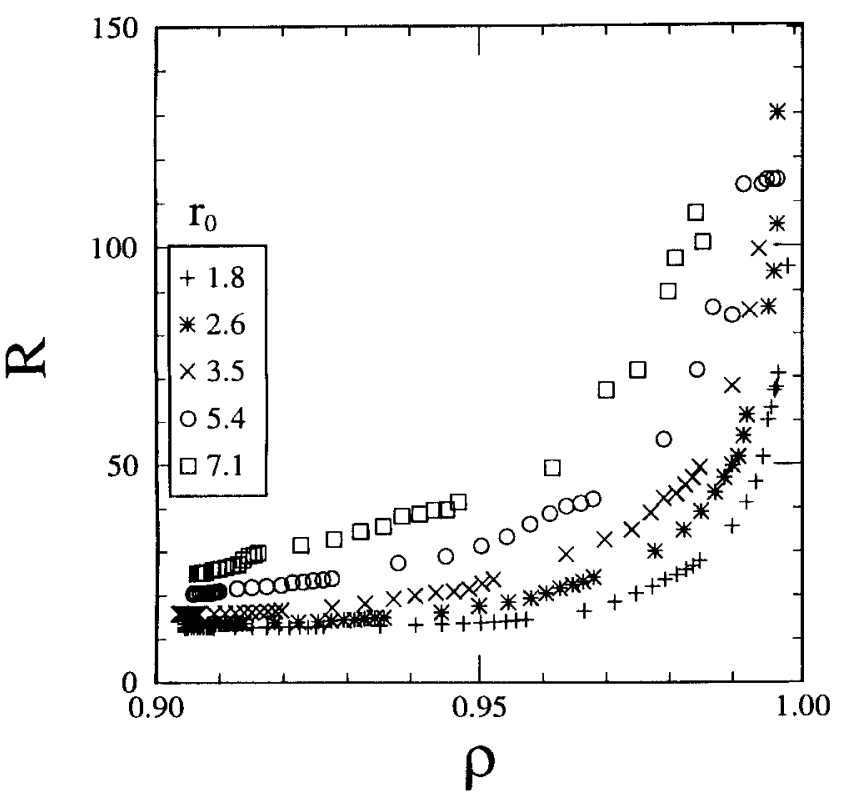

Fig. 5. Dependence of the mean grain size on the density $(\rho=1-\Phi)$ for a series of initial pore sizes.

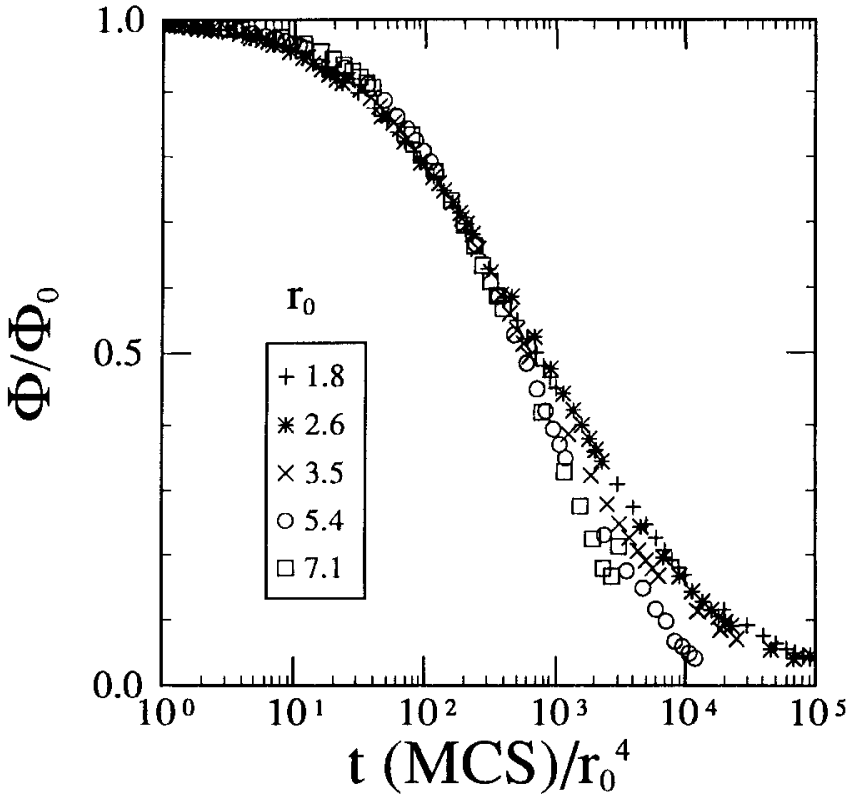

Fig. 6. Dependence of the porosity evolution kinetics on initial pore size, where porosity has been normalized by its initial value and time has been normalized by $r_{0}^{4}$.

"lifetime" than those of smaller pores. Note that, on a $\log$ $r-\log t$ scale, the decay of a pore is fairly abrupt, which justifies our use of the term "lifetime" in describing its kinetics.

According to the pore-shrinkage equation, derived for a regular pore array in part $I$, the pore size should decrease with time following a cubic law, i.e.

$$
r^{3}=r(0)^{3}-\frac{C D_{g b} t}{\lambda}
$$

where $D_{g b}$ is the grain-boundary diffusivity, $\lambda$ is the initial pore spacing, and $C$ is a constant. Motivated by this prediction (which is strictly valid if pores are identical in size and form a periodic array) we replot the individual pore data (see Fig. 9) in the form of $r^{3}$ vs $t$ in Fig. 10. Because of the large difference in the pore lifetimes, it is difficult to clearly repre-

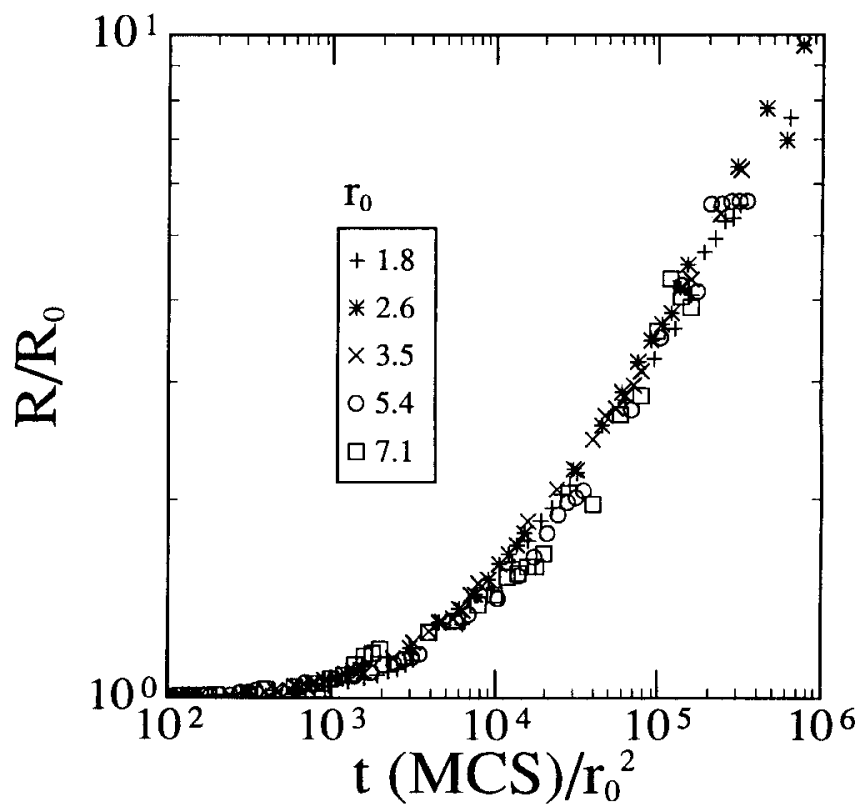

Fig. 7. Dependence of mean grain-size evolution kinetics on initial pore size, where grain size has been normalized by its initial value and time has been normalized by the initial pore area. 


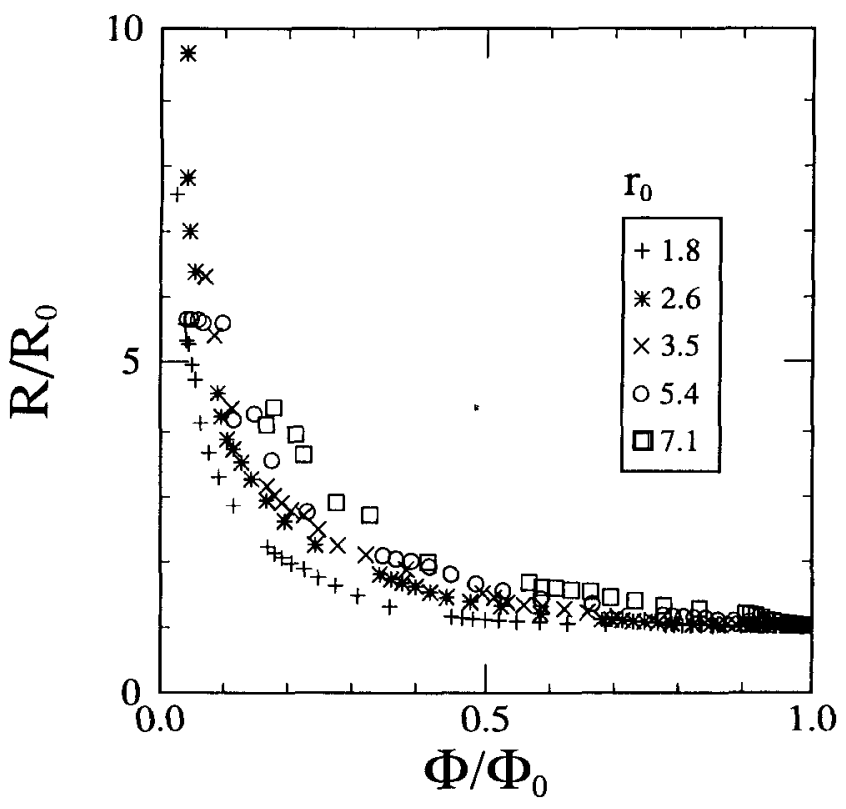

Fig. 8. Dependence on the instantaneous mean grain size (normalized by its initial value) on the instantaneous porosity (normalized by its initial value) for a series of initial pore sizes.

sent all the data on the same scale. Also, the statistical fluctuations for these individual pore data are inherently larger. Nevertheless, all pores, regardless of initial size, do seem to decay at approximately the same rate in $r^{3}$, and the curves connecting the data in Fig. 10 appear to be slightly concave upward. The latter feature is expected from Eq. (1) because, in the present simulation, the pore spacing may increase with time.

Although the sizes of all pores decay, some grains grow while other shrink. Despite occasional fluctuations, they generally follow a basic trend that larger grains tend to grow while smaller grains shrink. This, of course, is expected from the theory of grain growth. ${ }^{5}$

We have already demonstrated that the average grain size increases monotonically with time during final-stage sinter-

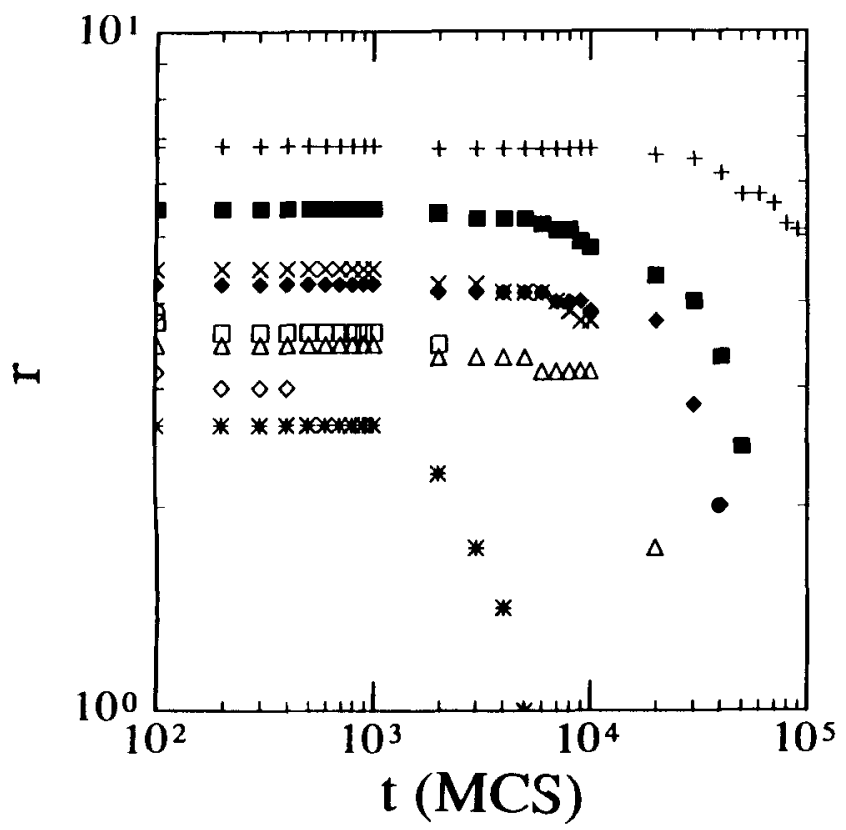

Fig. 9. Temporal evolution of the size of eight individual pores during the course of a single, final-stage sintering simulation.

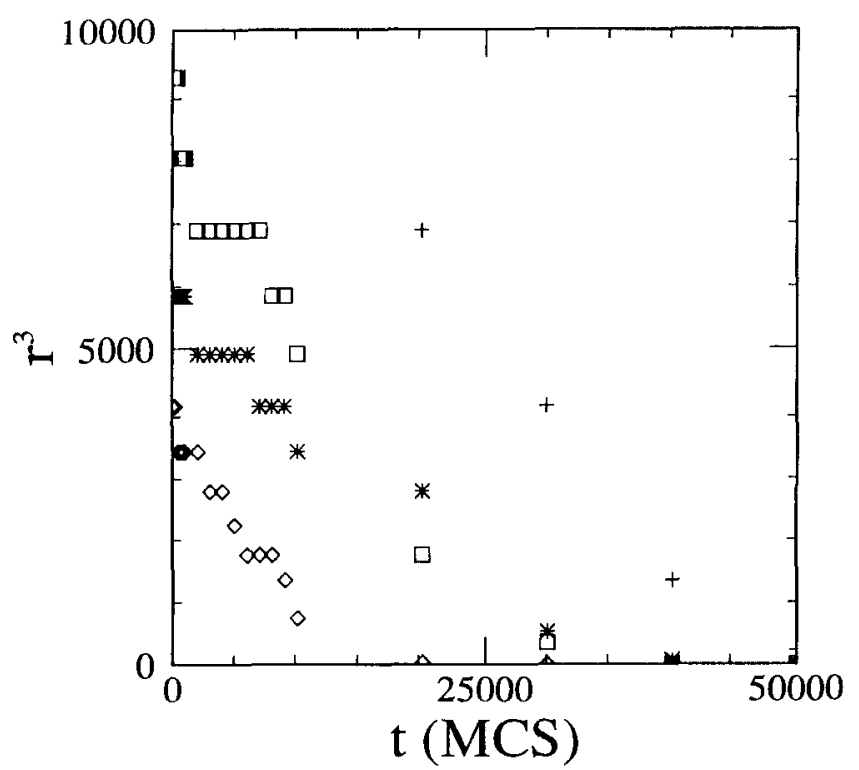

Fig. 10. Temporal evolution of the cube of the size of four individual pores.

ing. The average pore size was found to increase as well, but only by a small amount and very briefly. This is due to both a small number of pore coalescence events and to the more rapid shrinking of small pores compared to large pores. To contrast the relative behavior of pores and grains, we have plotted the average grain size versus average pore size for five different sets of initial conditions (Fig. 11). This plot clearly demonstrates that the average pore size initially decreases, then remains relatively unchanged throughout the remainder of the simulation apart from occasional statistical fluctuations. Note that, since the absolute amount of coarsening during the initial stage of the simulation appears to be approximately the same for all of the microstructures, the fractional increase of the average pore size is larger for microstructures with smaller initial average pore size.

\section{(4) Grain-Boundary Pinning by Pores}

A careful examination of Figs. 1 and 2 suggests that pores, although mobile and shrinking, are rather effective in pin-

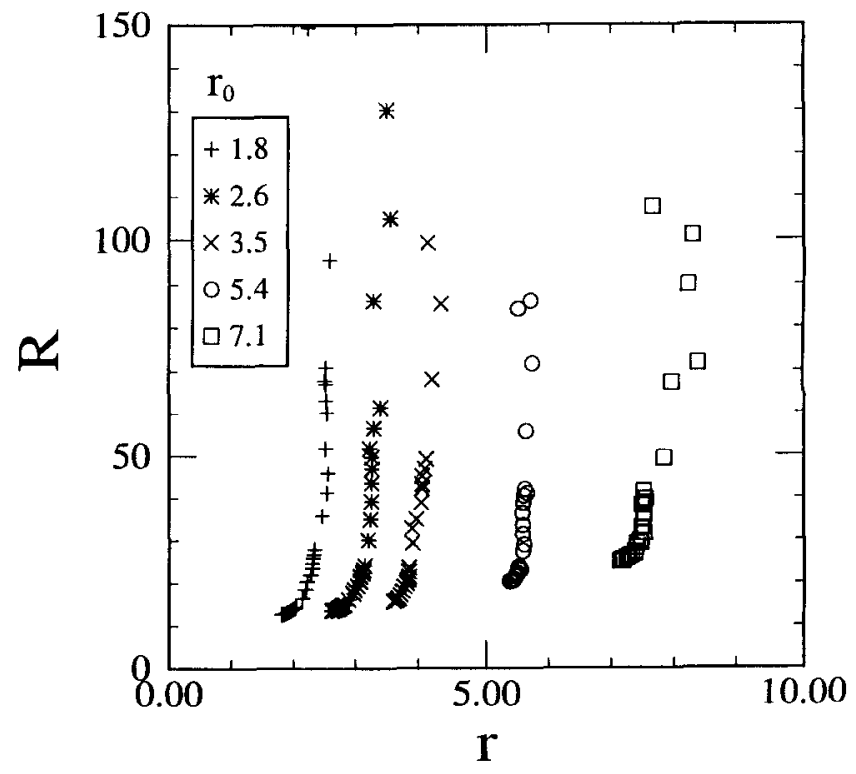

Fig. 11. Dependence of the mean instantaneous grain size on the mean instantaneous pore size for a series of inital pore sizes. 
ning grain boundaries. This is supported by several observations described below. First, in preparing the initial state for sintering simulations, we introduced pores by replacing a preselected fraction of the grains with pores and then allowed the microstructure to equilibrate without pore shrinkage. $\left(D_{g b}=0\right.$ but $D_{m} \neq 0$, in the notation of part I.) Invariably, we found that the microstructure reaches an asymptotic grain size; i.e., grain growth has stopped because of pore pinning. Second, by counting the number or pores per grain at different times during the sintering simulation, we find (as shown in Fig. 12) that the distribution of pores per grain remains approximately independent of time, even though the grain size has increased markedly. Indeed, since a typical grain has six boundaries (in two dimensions), it can be verified from Fig. 12 that every grain boundary has an average of one pore. Srolovitz et al. have shown by a computer simulation and a simple model of grain-growth stagnation by static particles, that the above geometrical relationship is precisely the condition required for pinning the triple points (i.e., the points at which three grain boundaries meet in two dimensions). ${ }^{6}$ Apparently, pores are capable of providing roughly the same pinning effect as stationary particles, even though they are both mobile and shrinking.

The geometrical relation referred to above predicts that, in two dimensions

$$
R=\sqrt{\frac{3}{\Phi}} r
$$

if the pinning is totally effective (e.g., with rigid, immobile particles), where $\Phi$ is the volume fraction of pinning particles. ${ }^{6}$ We compare our data with this theoretical relationship by plotting $(R / r) \sqrt{\Phi}$ vs $\Phi$ in Fig. 13. The theoretical prediction is also indicated in the plot by the horizontal dashed line at $\sqrt{3} \cong 1.73$. Data above the theoretical line (meaning faster grain growth than predicted) may be regarded as indicating less effective grain-boundary pinning pores compared to static particles. Based on this consideration, we find that smaller pores are not as effective, initially, as larger ones for grain-boundary pinning in a porous solid. However, we also find that their effectiveness improves with time as manifested by the decreasing value of $(R / r) \sqrt{\Phi}$ with decreasing $\Phi$. Indeed, recalling the higher fractional coarsening of the pore size associated with smaller pores discussed in Section III(3) (see Fig. 11), we may attribute this improvement in pinning effectiveness to the initial increase of the pore size. This interpretation is consistent with the trend exhibited in Fig. 13 because larger pores do not show any evidence of increased pinning effectiveness as $\Phi$ decreases. In this way, all the data converge to the same point (at $\Phi \approx 0.035$ for the present simulations).

Once all the data converge to the asymptotic $(R / r) \sqrt{\Phi}$ vs $\Phi$ curve because of pore coarsening, the underlying trend seems to be a gradual loss of pinning effectiveness with further decreases in porosity, $\Phi$. We believe that this simply reflects the fact that, as $\Phi$ approaches zero, the only remaining pores are in the interior of grains and, hence, are ineffective at retarding normal grain growth. Indeed, near $\Phi=0$, the prediction of Eq. (2) apparently breaks down entirely as $(R / r) \sqrt{\Phi}$ diverges. This is further supported by the data shown in Fig. 7 where the grain size gradually approaches the $\sqrt{t}$ dependence which is characteristic of normal grain growth. For example, Figs. 3 and 4 show that the onset of parabolic growth kinetics occurs near $t=10^{5} \operatorname{MCS}(\Phi=0.02)$ for $r_{0}=1.8$ and $t=2 \times 10^{6} \operatorname{MCS}(\Phi=0.04)$ for $r_{0}=7.1$.

This complicated interplay between pinning and normal growth is the cause for the relatively large scatter of data observed in Fig. 8. The branch with the smallest $r_{0}$ has the lowest $R / R_{0}$ because its $R_{0}$ is relatively larger (by a factor of 2 when normalized by $r_{0}$ at $\Phi=0.1$ according to Fig. 13) than the rest. Other than that, the data in Fig. 8 follow the expected $\sqrt{1 / \Phi}$ dependence until $\Phi$ becomes too small.

\section{Discussion}

\section{(1) Pore Size-Grain Size-Porosity Relationship}

Our results demonstrate that the pore size at the beginning of final-stage sintering plays a major role in controlling the development of the late-stage sintering microstructure. A smaller initial pore size results in a smaller grain size, both initially and at all subsequent stages at any given density. This is clear not only from the $R$ vs $\rho$ plot (Fig. 5) but also from the normalized $R / R_{0}$ vs $\Phi / \Phi_{0}$ plot (Fig. 8). These results can be understood if we recall that the average pore size remains essentially constant and that the pinning prediction, Eq. (2), is nearly obeyed. Thus, a smaller initial pore size will be carried through the entire sintering lifetime, resulting in a finer interpore spacing at any given density. Since, in two dimensions, grainboundary pinning is entirely determined by the pore (or par-

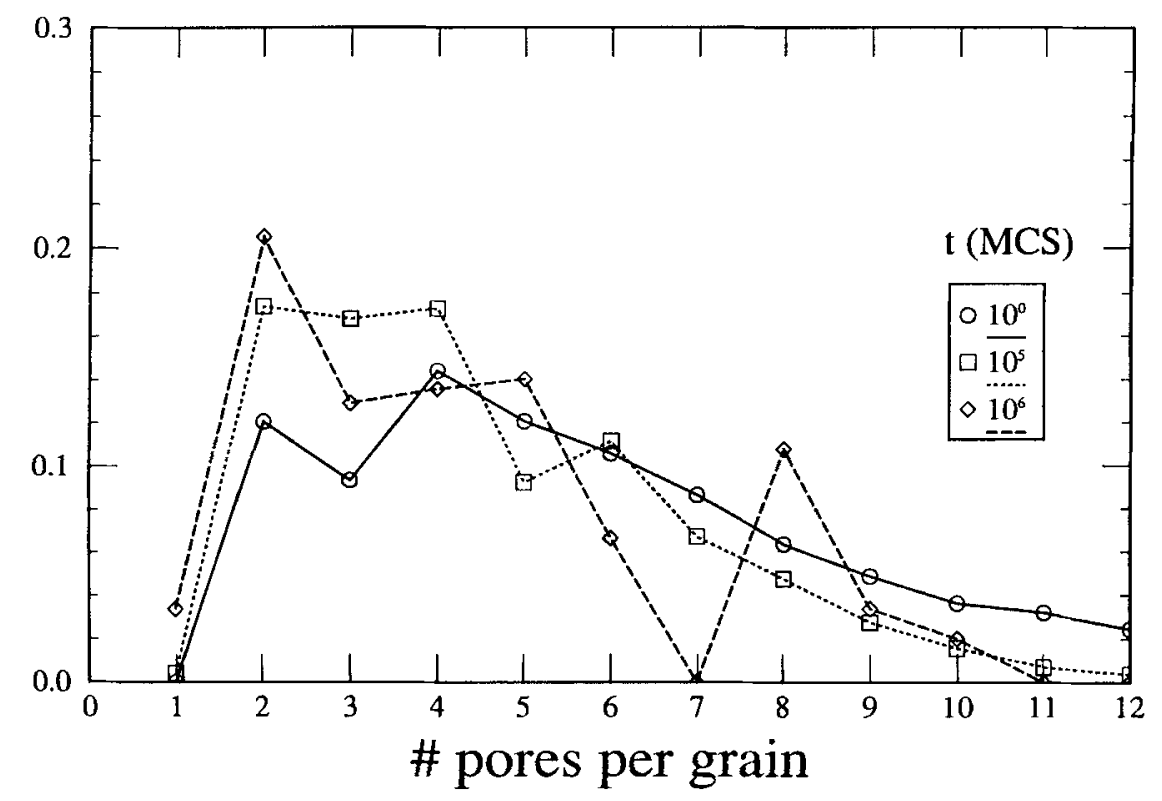

Fig. 12. Distribution of the number of pores per grain at different times during the course of a single, final-stage sintering simulation. 


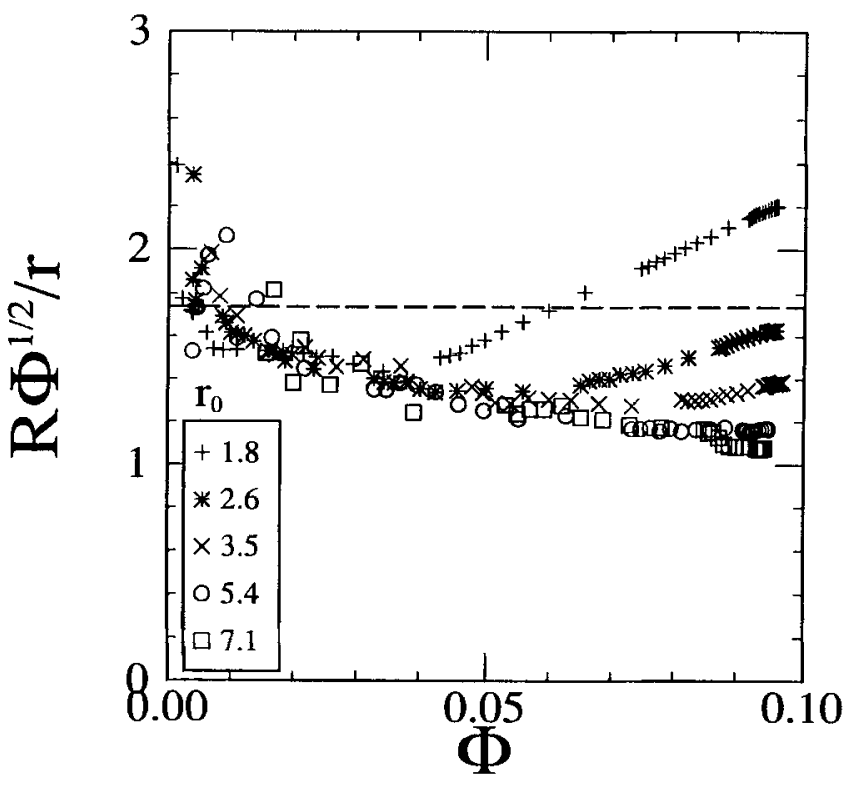

Fig. 13. Plot of the scaling function suggested by Eq. (2) for a series of simulations with different initial pore sizes.

ticle) spacing, ${ }^{6}$ this leads to a smaller grain size at all times. The additional benefit of a smaller pore size in reducing growth in terms of the normalized quantity $R / R_{0}$ is unexpected, but can be rationalized in terms of the increasing pinning effectiveness of smaller pores discussed in Section III (4).

\section{(2) Kinetics of Final-Stage Sintering}

The main kinetic findings of these simulations are summarized in Figs. 6 and 7 . Combined with the results obtained in part I, we may state that the sintering time scales with $r_{0}^{4} / D_{g b}$, and the grain-growth time scales with $r_{0}^{2} / D_{m}$. Thus, two scaling times are in operation concurrently. The origin of these scaling times can be understood from a dimensional analysis of the governing equations of pore shrinkage and grain growth.

The pore shrinkage equation, (Eq. (1)), which is exact for pores of an identical size in a one-dimensional periodic array, can be rewritten as

$$
\left(\frac{r}{r_{0}}\right)^{3}=1-C\left(\frac{D_{g s} t}{r_{0}^{3} \lambda_{0}}\right)
$$

Now, referring to the more realistic final-stage sintering microstructure, we note that the initial pore spacing, $\lambda_{0}$, is of the order of $R_{0}$, according to the observation of Fig. 12 that every grain boundary has an average of one pore. Further note that $R_{0}$ is related to $r_{0}$ by Eq. (2), because of grainboundary pinning by the pores. Thus, the mean pore lifetime is proportional to $r_{0}^{4} / D_{g b}$, which is consistent with the scaling relation for sintering (Fig. 6).

The normal grain-growth equation ${ }^{5,7}$

$$
R^{2}=R_{0}^{2}+C^{\prime} D_{m} t
$$

( $C^{\prime}$ is a constant) may be rewritten in a form similar to that for the evolution of pore size (Eq. (3))

$$
\left(\frac{R}{R_{0}}\right)^{2}=1+C^{\prime}\left(\frac{D_{m} t}{R_{0}^{2}}\right)
$$

Again noting that $\boldsymbol{R}_{\mathbf{0}}$ is related to $\boldsymbol{r}_{0}$ by Eq. (2), we find that the time scale for grain growth may be described by $r_{0}^{2} / D_{m}$. This is consistent with the scaling relation for grain growth found in the simulations after long times (Fig. 7). Note that the parabolic grain-growth law, Eq. (5), holds only if the pore drag is small, which is not the case at the beginning of finalstage sintering. Indeed, grain-size evolution is governed by a combination of Eqs. (2) and (3), which can be shown to yield grain-growth kinetics slower than $\sqrt{t}$. Therefore, the graingrow th kinetics can be divided into two regimes: (1) an initial period of very slow pore-limited growth and (2) a period when grain growth is only slightly impeded by the pores (this after a long time). For example, we find that a decrease in $\Phi / \Phi_{0}$ from 1 to 0.3 is accompanied by only a factor of 2 increase in grain size. Thus, the nearly normal grain-growth regime occurs after a long period over which little growth occurs; i.e., the grain-growth kinetics may be described as normal grain growth preceded by an incubation time. Referring to both Figs. 8 and 13 , we find that parabolic grain growth occurs when $\Phi<0.03$ (i.e., when the pinning relation begins to break down).

The above dimensional analysis of the shrinkage and grain-growth equations serves only as a qualitative rationale for the scaling relations observed. A more rigorous analysis, taking into account the entire population or pores and grains of different sizes, is required to substantiate the argument. We have recently conducted such an analysis and essentially confirmed the above finding. This analysis will be published elsewhere. ${ }^{8}$

\section{(3) Implications}

One of the criteria proposed in the literature for evaluation of the quality of sintering is based on the grain size-density relationship. ${ }^{9}$ In general, it is thought that slower grain growth during densification is desirable. Figure 5 shows that a smaller initial pore size can produce the desired effect. If one further recognizes that the densification is governed by a time scale of $r_{0}^{4} / D_{g b}$ and grain growth is governed by a time scale of $r_{0}^{2} / D_{m}$, then it is obvious that a small initial pore size, a high grainboundary diffusivity, and a low grain-boundary mobility are desirable. Note that, in a well-packed compact, the finalstage sintering begins when a Rayleigh instability condition is met (see part I) at $R_{0} / r_{0} \approx 2 \pi$ which corresponds to $\Phi_{0} \approx$ 0.09 . Further, there is little grain coarsening during initialand intermediate-stage sintering if good packing is first obtained. ${ }^{10-12}$ We believe that it is in this connection that a fine powder size and good green-state processing can exert a direct beneficial influence on the quality of final-stage sintering.

The most surprising findings of the present simulation is that the average pore size remains largely unchanged as the porosity decreases from 0.09 to 0.01 or less. Apparently, the dynamics in pore shrinkage is such that a self-similar poresize distribution is maintained. We recently were able to prove that such self-similar distributions are a rather general feature of the continuity equation for the pore-size distribution in the absence of pore coalescence. ${ }^{8}$ Many previous finalstage sintering theories have assumed geometric similitude, which in turn, led to a mean pore size that scales with the (growing) grain size. ${ }^{13-15}$ In other theories, the average pore size was found to decrease with time because all pores were assumed to be the same size. ${ }^{1}$ We have not found support for either of the above two assumptions in our simulations. (Experimental data on pore sizes and grain sizes in $\mathrm{Al}_{2} \mathrm{O}_{3}$ seem to suggest a much slower coarsening rate for the pores relative to that for the grains. ${ }^{16}$ )

Unfortunately, we are not aware of any detailed kinetic and microstructural studies of final-stage sintering to make a meaningful comparison with the current simulations. Most experimental observations in the past failed to distinguish between the intermediate and final stages of sintering or were only focused on specific features, such as pore-boundary separation. In view of the above discussion, we suggest that a direct comparison of the present simulations and the experimental observations may proceed in the following way. To focus attention on final-stage sintering, it is necessary to select a series of well-packed green bodies prepared with different powder particle sizes and sinter them first so that only 
closed pores of various mean sizes remain. Excessive grain coarsening should be avoided at this stage. ${ }^{10-12}$ Their microstructures then provide the appropriate initial state for the study of final-stage sintering. During subsequent densification, microstructural evolution should be monitored to examine the three major predictions of the present work: (1) the scaling relationship between $\Phi / \Phi_{0}$ and $r_{0}^{4} / D_{g b} ;(2)$ the scaling relationship between $R / R_{0}$ and $r_{0}^{2} D_{m}$; and (3) the time invariance of the average pore radius. However, the following caution on the limitations of the two-dimensional simulations should be noted first.

\section{(4) Limitations of the Two-Dimensional Simulations}

Limitations of two-dimensional simulations for predicting three-dimensional behavior primarily come from the different forms of the governing equations in two dimensions and three dimensions, and the different geometric relations between microstructural parameters and average (e.g., $R, r$, and $\lambda)$ or cumulative parameters (e.g., $\Phi$ ). Many of the above aspects can influence the competition between growth mechanisms and the overall kinetics of microstructural evolution. The most critical ones among these, in our view, lie in the pore-boundary interactions.

The grain-size pinning condition given by Eq. (2) is only valid in two dimensions. In recent three-dimensional Monte Carlo simulations of grain growth, stagnation in the presence of static particles strongly suggests that $R \propto r / \Phi^{1 / 3} .^{17}$ This result is analogous to the two-dimensional result (Eq. (2)) in that the important quantity is the ratio of the grain size to the mean spacing between pores. Although the constant of proportionality is known in two-dimensions (i.e., $\sqrt{3}$ ), it appears to be substantially smaller in three-dimensions and has not yet been predicted theoretically. Notwithstanding the present two-dimensional final-stage sintering simulations and the recent three-dimensional particle-limited grain-growth simulations, ${ }^{17}$ it remains unclear how all of the kinetic features of sintering (pore-shape evolution, grain-boundary diffusion, grain growth, and pore retardation of grain growth) are coupled. For this reason, it is imperative to extend the present simulations to three dimensions.

\section{Conclusions}

A two-dimensional Monte Carlo simulation procedure has been used to investigate the effect of initial pore size on the microstructural evolution and kinetics of final-stage sintering. Pores are found to effectively pin the grain boundaries from the beginning of final-stage sintering at a porosity of $\Phi=0.09$ until $\Phi \leq 0.03$. For $\Phi=0.03$, the remaining pores do not effectively retard grain-boundary migration and nor- mal grain growth occurs. Small pores were found to be less effective at retarding grain growth than expected on the basis of a simple grain-growth pinning model. The mean pore size was found to be nearly constant throughout the simulations.

The sintering time scales with $r_{0}^{4} / D_{g b}$ and the grain-growth time scales with $r_{0}^{2} / D_{m}$. Thus, a small initial pore size, a high grain-boundary diffusivity, and a low grain-boundary mobility are desirable to achieve rapid densification with little coarsening. Since the initial grain size and pore size at the onset of final-stage sintering are proportional to the initial powder size in a well-packed green body, the latter processing variable can exert a direct beneficial influence on the quality of the sintered product.

\section{References}

'G. N. Hassold, I-W. Chen, and D. J. Srolovitz, "Computer Simulation of Final-Stage Sintering: I, Model, Kinetics, and Microstructure," J. $\mathrm{Am}$. Ceram. Soc., 73 [10] 2857-64 (1990).

${ }^{2}$ R. L. Coble, "Sintering Crystalline Solids. I. Intermediate and Final State Diffusion Models," and "II. Experimental Test of Diffusion Models in Powder Compacts," J. Appl. Phys., 32 [5] 787-99 (1961).

${ }^{3}$ F. A. Nichols and W.W. Mullins, "Surface-(Interface) and Volume-Diffusion Contributions to Morphological Changes Driven by Capillarity," Trans. TMS-AIME, 233 [10] 1840-48 (1965).

${ }^{4}$ M. P. Anderson, D. J. Srolovitz, G. S. Grest, and P.S. Sahni, "Computer Simulation of Grain Growth I. Kinetics," Acta Metall., 32 [5] 783-91 (1984).

${ }^{5}$ M. Hillert, "Theory of Normal and Abnormal Grain Growth," Acta Metall., 13 [3] 227-38 (1965).

${ }^{6}$ D. J. Srolovitz, M. P. Anderson, G. S. Grest, and P.S. Sahni, "Computer Simulation of Grain Growth III. Influence of a Particle Dispersion," Acta Metall., 32 [9] 1429-38 (1984).

7J. E. Burke, "Role of Grain Boundaries in Sintering," J. Am. Ceram. Soc., 40, 80-85 (1957).

${ }^{8}$ I-W. Chen, "A Theory of Final-Stage Sintering"; unpublished work.

${ }^{9}$ M. P. Harmer, "Use of Solid-Solution Additives in Ceramic Processing"; pp. 679-96 in Advances in Ceramics, Vol. 10, Structure and Properties of $\mathrm{MgO}$ and $\mathrm{Al}_{2} \mathrm{O}_{3}$ Ceramics. Edited by W. D. Kingery. American Ceramic Society, Columbus, $\mathrm{OH}, 1984$.

${ }^{10}$ T-S. Yeh and M. D. Sacks, "Effect of Green Microstructure on Sintering of Alumina"; pp. 309-31 in Ceramic Transactions, Vol. 7, Sintering of Advanced Transactions. Edited by C. A. Handwerker, J. E. Blendell, and W. A. Keyser. American Ceramic Society, Westerville, OH, 1990.

${ }^{11}$ C. P. Cameron and R. Raj, "Grain-Growth Transition during Sintering of Colloidally Prepared Alumina Powder Compacts," J. Am. Ceram. Soc., 71 [12] 1031-35 (1988).

${ }^{12}$ D. J. Sordelet and M. A. King, "Sintering of Monosized, Spherical Yttria Powders," J. Am. Ceram. Soc., 71 [12] 1148-53 (1988).

${ }^{13}$ W. D. Kingery and B. Francois, "Grain Growth in Porous Compacts," J. Am. Ceram. Soc., 48 [10] 546-47 (1965).

${ }^{14}$ R. J. Brook, "Pore-Grain Boundary Interactions and Grain Growth," J. Am. Ceram. Soc., 52 [1] 56-67 (1969).

${ }^{15}$ R. J. Brook, "Controlled Grain Growth"; pp. 331-64 in Treatise on Materials Science and Technology, Vol. 9. Edited by F. F.Y. Wang. Academic Press, New York, 1976.

${ }^{16} \mathrm{~A}$. Mocellin and W. D. Kingery, "Microstructual Changes during Heat Treatment of Sintered $\mathrm{Al}_{2} \mathrm{O}_{3}$," J. Am. Ceram. Soc., 56 [6] 309-14 (1973).

${ }^{17}$ M. P. Anderson, G.S. Grest, R. D. Doherty, K. Li, and D. J. Srolovitz, "Inhibition of Grain Growth by Second Phase Particles: Three-Dimensional Monte-Carlo Computer Simulations," Scr. Metall., 23, 753-58 (1989). 